\title{
Changes in leaf water use after removal of leaf lower surface hairs on Mallotus macrostachyus (Euphorbiaceae) in a tropical secondary forest in Malaysia.
}

\begin{abstract}
Leaf hairs may assist in maintaining high leaf water use efficiency in tropical secondary forest tree species. We compared leaf temperature, transpiration, photosynthesis and water use efficiency between hairy and depilated leaves in Mallotus macrostachyus (Euphorbiaceae), to determine the role of leaf hair in leaf water use efficiency (WUE) in tropical degraded secondary forest in Malaysia. Measurements were made on five mature individuals growing in sun-exposed conditions and five in shaded conditions. The hair dry weight per unit leaf area was significantly greater in sun leaves than in shade leaves. The transpiration rate (Trmax) of depilated leaves in sun-exposed conditions was slightly higher than in hairy leaves in both morning and afternoon measurements. In contrast, Trmax in the shade leaves was almost identical in hairy and depilated leaves. Leaf stomatal conductance (g s) in the morning showed almost the same value among leaf types and light conditions. In the afternoon, g s slightly decreased from the morning values in both sun and shade conditions. In the morning, the leaf water use efficiency (A max/Trmax) in both conditions did not differ significantly between hairy and depilated leaves. However, in the afternoon, WUE in the depilated leaves was significantly lower than in hairy leaves in sun-exposed conditions. These observations suggest that leaf hairs in M. macrostachyus contribute to the high leaf water use efficiency in drought conditions, such as high vapor pressure deficit experienced at midday in degraded tropical secondary forests.
\end{abstract}

Keyword: Boundary layer; Leaf trichomes; Photosynthesis; Transpiration; Water use efficiency. 